\title{
A Low-cost Webcam-based Eye Tracker and Saccade Measurement System
}

\author{
Ahmad Aljaafreh, Murad Alaqtash, Naeem Al-Oudat, Jafar Abukhait, and Ma'en Saleh
}

\begin{abstract}
Eye movements are integrated with cognitive processes, which indeed make it a helpful research basis for the investigation of human practices. Eye movements can be deployed in discovering several cognitive processes of the brain. This research utilizes low-resolution webcam to develop an eye tracker and saccades measurement tool to extensively lower the gadgets expenses. A consistent algorithm is developed to suit the quality of the webcam using open-source software (Python) to record the time series of the eye location. Likewise, several algorithms are proposed to extract high-level eye movement saccadic measurements from the raw gaze outputs. A pilot study is performed on ten normal participants and Multiple Sclerosis (MS) patients. Experimental results demonstrate that the proposed system is quick, simple and efficient for eye tracking and saccade measurement. The developed tool can be used by clinicians and medical physicians for the diagnosis and identification of neurological disorders.
\end{abstract}

Keywords - Eye-movements, Eye-tracker, Saccades parameters, Saccade latency, Amplitude gain, Peak velocity, Neurological disorders, Multiple Sclerosis.

\section{INTRODUCTION}

$\mathrm{R}^{\mathrm{e}}$ ecently, eye tracking has been addressed by the community to give an insight into brain cognitive processes. It has been utilized to study human behaviors in different fields, for instance, driving [1], image scanning [2], arithmetic [3], analogy [4], human-computer interaction [5], and reading [6]. In this context, eye-tracking and dynamic stimuli are analyzed. Dynamic stimuli refer to the moving objects (target) that the user is looking at which is either in the environment or on a computer screen in a stationary setup. During recent years, the interest in using dynamic stimuli has grown, both for stationary and mobile eye-tracking. Normally, researchers analyze eye movements in terms of fixations and saccades.

Fixations are the pauses over informative regions of interest while saccades are rapid movements between fixations. Saccade is a quick, simultaneous movement of both eyes between two or more phases of fixation in the same direction.

The authors are with the Communications, Electronics, and Computer Engineering Department, Faculty of Engineering, Tafila Technical University, Tafila, Jordan. (Corresponding author; Ahmad Aljaafreh; email: a.aljaafreh@ttu.edu.jo)
Saccadic velocities, amplitudes, latency, and duration are the most common analysis metrics for studying human behavior. Latency is the time taken from the appearance of a target to the beginning of a saccade in response to that target. Amplitude is the size of the saccade, usually measured in degrees or arc minutes. Amplitude determines the saccade accuracy. This is sometimes denoted using "gain". The gain is the ratio of the actual saccade amplitude divided by the desired saccade amplitude. Peak velocity is the highest velocity reached during the saccade as shown in Fig. 1.

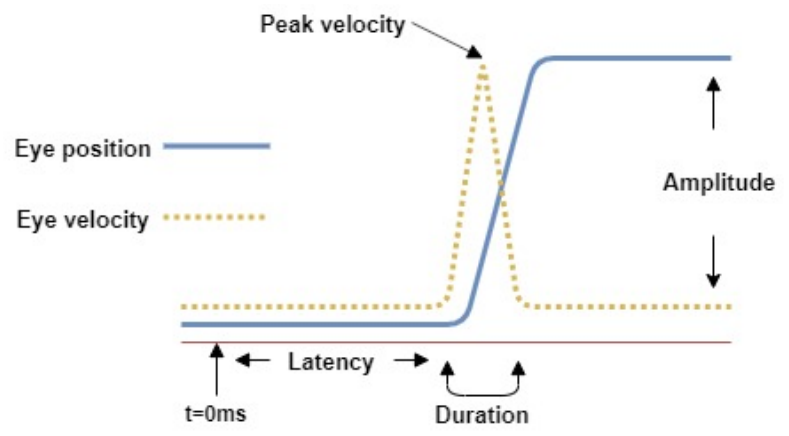

Fig. 1 Saccadic parameters [7]

The fourth saccade parameter is the duration which is the time taken to complete the saccade. Saccadic measurements have been used in various research domains such as deficient saccadic function being used to understand cognitive [8] or visual [9] processes, neurological inspection and diagnosis [10] or identifying practical shortfalls in everyday tasks $[11,12]$.

Recently, real-time computer vision algorithms have been deployed to detect the eyeball movement from a digital image sequence. Movement detection is utilized for fixation and saccadic gaze movement identification. Infrared cameras are widely used in the on-shelf commercial eye tracker [13]. The point, at which someone is looking, is located on the screen based on the relative locations of the pupil center in the video image of the eye. Video cameras are also used in commercial eye trackers. Webcam-based gaze tracker has less accuracy and cost than infrared-based eye trackers [14]. OpenCV library is exploited by most webcam-based systems to detect face and gaze position based on the relative position of the pupil within the standard geometry of eyes. The major bottleneck for eyetrackers is the algorithms that analyze the recorded eyetracking signals. Analysis of eye-tracking data has many 
challenges including building flexible algorithms for a highly noisy signal. Recorded eye-tracking signals may have different types of noise and disturbances resulted from the different types of eye-trackers $[15,16]$ and individual differences between the users.

In this work, several algorithms are proposed: extracting high-level eye movement saccadic measurements from the raw gaze outputs; developing a dynamic stimuli application using python; and eye-tracking based on low-resolution webcam video by recording the raw data of gaze location and dynamic stimuli. To validate the proposed tool, a comparison of saccadic measurements is performed for normal volunteers and Multiple Sclerosis (MS) patients. Several studies employ MS patients to validate their tools for the identification and diagnosis of neurological disorders [17, 18]. Some relevant studies can be found in [19-23].

The rest of this paper is organized as follows. Section II details the methodology used in this research. Results are shown and discussed in Section III. Section IV concludes and summarizes the work.

\section{Methodology}

In this section, the system hardware and software setup are described in details. Software flow diagram, shown in Fig. 2, represents the data acquisition and processing steps required to measure the saccadic parameters. The software was written in python 3.6 using JetBrains PyCharm IDE. The source code is available online at [24].

\section{A. Experimental Setup}

Experiments were conducted using an HP EliteBook 8440p laptop with an Intel i7 core processor running Microsoft Windows 10 Pro operating system. A built-in webcam that operates at $30 \mathrm{fps}$ was used. Participants were seated at $70 \mathrm{~cm}$, eye-monitor distance, in front of 14-inch size and 1600x900pixel resolution monitor. A fixator was used to stabilize the chin of participants during experiments and appropriate lighting of the room was guaranteed to ensure proper video capturing. Participants were trained prior to experimentation to make sure that they keep their eyes on the dynamic stimulus without anticipating the pattern of the movement.

\section{B. Data Acquisition}

When Data acquisition is a real-time process that records time series for both eye movement, i.e. the relative coordinates of pupils, and dynamic stimulus. In this work, a simple dynamic stimulus, as a white circle on a black screen, was designed. The circle appears in two different locations along the horizontal axes of the screen as in Fig. 3. An experiment of 200 seconds long was recoded for each participant. The experiment includes a number of trials. Each trial represents a sequence of actions to display the marker, measure and record the data. The marker stays for a random time in each location to prevent anticipating of the next location by the participant.

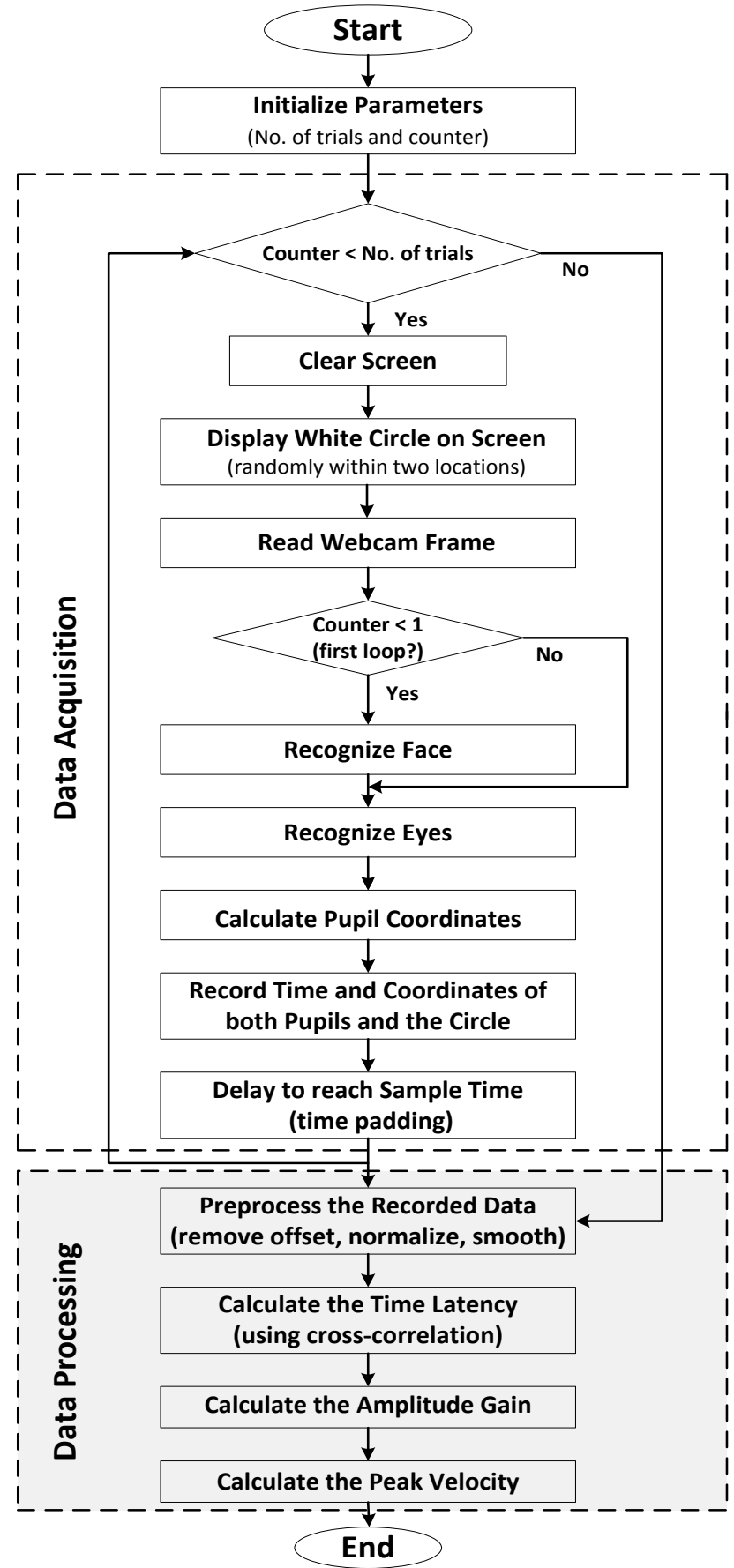

Fig. 2 System flow diagram representing data acquisition and processing steps to measure the saccadic parameters
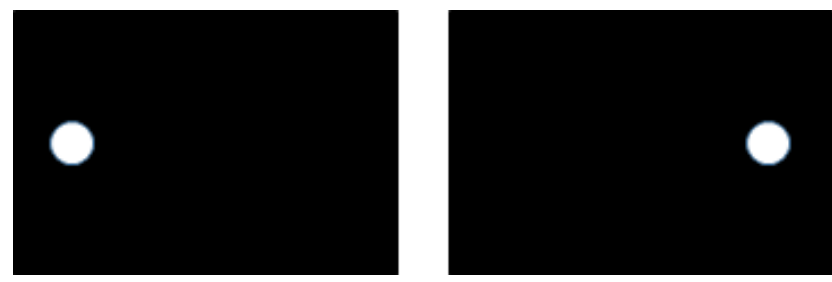

Fig. 3 The dynamic stimulus represented as a white circle on a black screen for two different locations on the screen (Left and Right) 
Pygame, Dlib and OpenCV software packages are used for face, eye, and pupil detection. The time and location of both pupils and marker are saved in a file for processing. Fig. 4 shows an example of the pupil and circle location sequences. The noisy series is for the pupil while the smoothed one is for the circle. The process of saccades measurement starts after recording the time series of the pupil and circle locations.

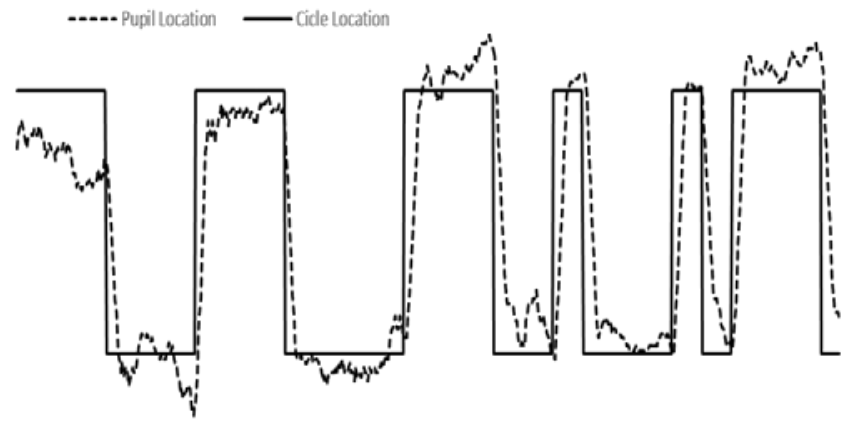

Fig. 4 The location sequences of the pupil and marker along time line.

\section{Data Processing and Analysis}

Data processing stage starts after acquiring and recording the time sequence of eye pupils and the dynamic stimulus locations. In this stage, the saccadic parameters (time latency, amplitude gain and peak velocity) are calculated from the raw data as described in the following:

\section{1) Time Series Processing}

The time series represents the time and the location for both pupils and stimulus marker. This series is recorded during the experiment and preprocessed as follows. First, the time series of the pupil location is normalized and the DC offset is removed. This process is performed by subtracting the mean and dividing by the standard deviation of the whole series. After normalization, the series is filtered using a low pass filter (moving average with 10 samples window length) to smoothen the signal. Then the time series is ready to be processed for calculating the saccades' parameters.

\section{2) Saccadic Latency}

Saccade latency is the time taken from the appearance of a target to the beginning of a saccade response to the white circle movement (dynamic stimulus). Saccade latency represents the delay time between the two recorded time series (the time series of the pupil and the time series of the white circle). The time shift (delay) between the two-time series is shown in Fig. 3. Cross-correlation is used to find the delay between the two sequences and thus; finding the latency of the eye movement for both eyes. The maximum value of the crosscorrelation determines the point of maximum delay.

\section{3) Saccadic Peak Velocity}

Saccades peak velocity is the highest velocity reached during the saccade. Since the time series is a noisy signal; a robust algorithm is developed to measure the peak velocity. The steps of the algorithm are as follows:

- Calculating the zero crossings for the eye pupil preprocessed time series. Zero crossings are the points where the gaze changes sign.

- Calculating the zero crossings for the dynamic stimulus (circle) time series.

- Finding the index of the zero-crossing points of the circle time series.

- Between every two zero-crossing points of the circle time series, there should be one zero-crossing point of the pupil time series. Loops the zero-crossing signal of the pupil location looking for the points were only one point indexed in between the consequent points in the circle zero-crossing series.

- The maximum slope is calculated around these points

- The saccadic peak velocity is calculated as the average of the maximum slope is calculated for all points.

\section{Saccadic Amplitude Gain}

Amplitude gain is the ratio between the desired angle that the eye should move and the actual angle of the eye. The actual angle of the eye pupil is calculated from the captured frame. The number of pixels, that the pupils moved in the frame, is measured and converted to distance based on the focal length of the eye. The desired angle is calculated from the moving distance of the circle and the distance between the eye pupil and the screen. The algorithm steps for calculating the saccades amplitude gain are:

- Calculate the circle distance from the center of the screen to the most left or right location in pixel and convert it to distance $\left(C_{d}\right)$ in mm depends on screen specifications.

- Calculate the maximum angle of the circle $\left(C_{a}\right.$ ) from the center of the screen to the most left / right location, as in Equ. 1, assuming the distance between the eye pupil and the screen $\left(E_{d}\right)$ is fixed.

$C_{a}=\tan ^{-1}\left(\frac{C_{d}}{E_{d}}\right)$

- Calculate the distance of the eye pupil in pixel $\left(P_{p}\right)$ from center to the most right / left and convert it to distance $\left(P_{d}\right)$ unit $(\mathrm{mm})$ as in Equ. 2.

$P_{d}=\frac{P_{p} * E_{d}}{C_{f}}$

Where $\left(C_{f}\right)$, is the focal length of the camera, which is measured as described later in Camera Focal Length Calculation section. 
- Calculate the angle of the pupil ( $P_{a}$ ), the angle represents the movement of pupil while tracking the dynamic stimulus form one location to the other, as in Equ. 3.

$$
P_{a}=\tan ^{-1}\left(\frac{P_{d}}{E_{f}}\right)
$$

Where $\left(E_{f}\right)$, is the focal length of the human eye (eye diameter), typical is $24 \mathrm{~mm}$.

- Calculate the amplitude Gain $\left(A_{g}\right)$ as the ratio between the eye angle $\left(E_{a}\right)$ and the circle angle $\left(C_{a}\right)$ as in Equ. 4.

$$
A_{g}=\frac{E_{a}}{C_{a}}
$$

\section{E. Camera Focal Length Calculation}

The focal length of the camera is the distance between the lens and the image sensor. Saccade measurement is dependent on camera focal length and screen resolution. The proposed saccade measurement widget is independent of screen size and webcam type. It can be used on any laptop without any need for modification of the code. Tkinter python package was used to calculate screen pixel density. A marker or object with a known width $(W)$ is placed in a well-determined distance ( $D$ ) from the camera. The height of captured object in the image is defined as $(H)$. The focal length $\left(F_{l}\right)$ of our camera, illustrated in Fig. 5, is calculated as in Equ. 5 as:

$$
F_{l}=\frac{H^{*} D}{W}
$$

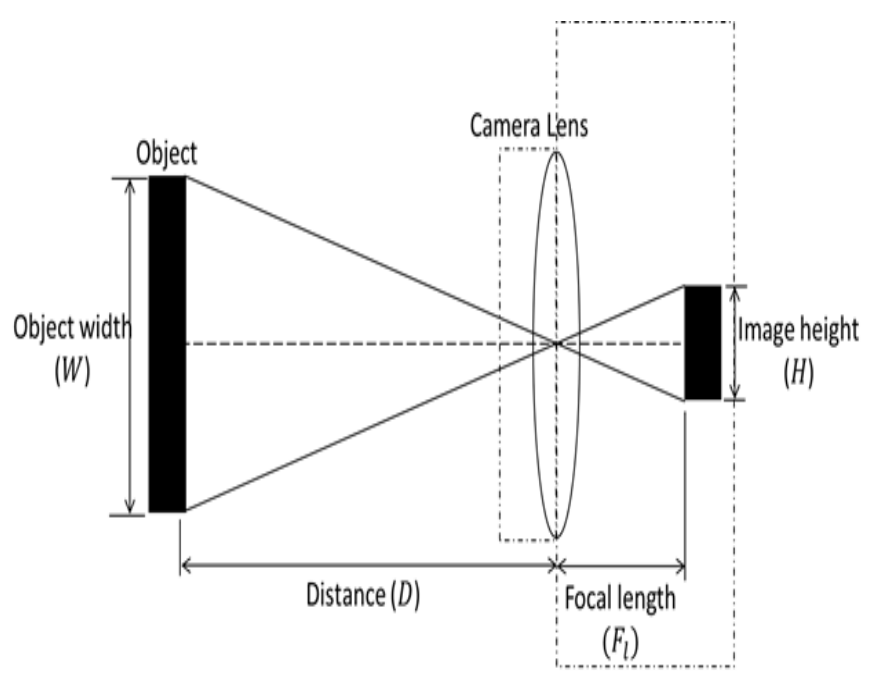

Fig. 5 Calculation of the focal length for the webcam

\section{RESULTS AND DISCUSSIONS}

In this section, we verified the correctness of the measurements taken by our tool, then we applied the same settings on 10 patients as a pilot study to further proof the concept and effectiveness of the tool. Discussion of the results were provided as well.

\section{A. Correctness of the Tool's Measurements}

Table I demonstrates the saccadic parameters; latency time, peak velocity, and amplitude gain, for two participants; 48year and 16-year old males, respectively. The mean and standard deviation (StdDev) were calculated for 10 different trials. The results showed that our measurements are within the normal range for both participants. To evaluate the precision and repeatability of our measurements; the coefficient of variation (CV) metric is used as a performance metric. $\mathrm{CV}$ is defined as the ratio of StdDev to the mean. When CV is close to zero the measurement is considered more accurate. The mean CV of latency time, peak velocity, and amplitude gain for the participants are $0.0478,0.300$, and 0.209 , respectively. Therefore, the measurements of the latency time is more accurate than the measurements of the both peak velocity and amplitude gain.

\begin{tabular}{|c|c|c|c|c|}
\hline Participant & $\begin{array}{l}\text { Exp. } \\
\text { No. }\end{array}$ & $\begin{array}{c}\text { Latency } \\
\text { Time (ms) }\end{array}$ & $\begin{array}{c}\text { Peak } \\
\text { Velocity } \\
(\mathrm{mm} / \mathrm{s})\end{array}$ & $\begin{array}{c}\text { Amplitude } \\
\text { Gain (\%) }\end{array}$ \\
\hline \multirow[t]{12}{*}{1} & 1 & 373 & 0.078 & 1.80 \\
\hline & 2 & 339 & 0.120 & 1.08 \\
\hline & 3 & 373 & 0.150 & 1.10 \\
\hline & 4 & 345 & 0.270 & 1.80 \\
\hline & 5 & 335 & 0.100 & 1.10 \\
\hline & 6 & 332 & 0.120 & 1.02 \\
\hline & 7 & 339 & 0.130 & 1.03 \\
\hline & 8 & 369 & 0.270 & 1.30 \\
\hline & 9 & 345 & 0.120 & 1.20 \\
\hline & 10 & 335 & 0.200 & 1.60 \\
\hline & Mean & 348 & 0.156 & 1.303 \\
\hline & StdDev & 16.54 & 0.068 & 0.312 \\
\hline \multirow[t]{12}{*}{2} & 1 & 360 & 0.09 & 0.85 \\
\hline & 2 & 389 & 0.1 & 0.86 \\
\hline & 3 & 348 & 0.14 & 0.56 \\
\hline & 4 & 352 & 0.14 & 0.68 \\
\hline & 5 & 370 & 0.11 & 0.86 \\
\hline & 6 & 384 & 0.1 & 0.86 \\
\hline & 7 & 342 & 0.14 & 0.56 \\
\hline & 8 & 350 & 0.12 & 0.68 \\
\hline & 9 & 340 & 0.13 & 0.59 \\
\hline & 10 & 348 & 0.14 & 0.7 \\
\hline & Mean & 358 & 0.121 & 0.720 \\
\hline & StdDev & 17.21 & 0.020 & 0.128 \\
\hline
\end{tabular}

Table 1. The saccadic parameters' measurements for the two participants with 10 different trials 


\section{B. Pilot Study}

The developed tool can be used to diagnose neurological disorders. A pilot study in this work was performed on 10 Multiple Sclerosis (MS) patients. For each participant, we measured the saccadic parameters, latency time and amplitude gain; and the average was calculated for three trials. To evaluate the severity of patient's condition; we measured the latency time and amplitude gain for 10 different normal participants as depicted in Table II. The mean and the standard deviation (StdDev) are calculated for the ten participants per each group.

Table 2. The average latency time and amplitude gain of three trials for ten normal participants and ten MS patients

\begin{tabular}{|c|c|c|c|}
\hline Participant & No. & $\begin{array}{c}\text { Latency Time } \\
\text { (ms) }\end{array}$ & $\begin{array}{c}\text { Amplitude Gain } \\
(\%)\end{array}$ \\
\hline \multirow[t]{12}{*}{ Normal } & 1 & 302 & 0.82 \\
\hline & 2 & 295 & 1.10 \\
\hline & 3 & 312 & 0.83 \\
\hline & 4 & 288 & 1.09 \\
\hline & 5 & 301 & 1.15 \\
\hline & 6 & 278 & 1.24 \\
\hline & 7 & 320 & 0.70 \\
\hline & 8 & 335 & 0.78 \\
\hline & 9 & 339 & 0.62 \\
\hline & 10 & 345 & 1.08 \\
\hline & Mean & 312 & 0.941 \\
\hline & StdDev & 22.73 & 0.214 \\
\hline \multirow[t]{12}{*}{ MS } & 1 & 636 & 0.50 \\
\hline & 2 & 437 & 1.40 \\
\hline & 3 & 386 & 0.83 \\
\hline & 4 & 403 & 1.14 \\
\hline & 5 & 235 & 1.15 \\
\hline & 6 & 235 & 1.24 \\
\hline & 7 & 538 & 0.30 \\
\hline & 8 & 369 & 0.58 \\
\hline & 9 & 302 & 1.62 \\
\hline & 10 & 369 & 2.08 \\
\hline & Mean & 391 & 1.084 \\
\hline & StdDev & 125.38 & 0.546 \\
\hline
\end{tabular}

\section{Discussion}

The results demonstrate a significant difference between normal and MS participants in the mean of latency time parameter. In addition, there is a noteworthy variance for MS patients due to the variability and severity of disorders. Most of MS patients, 9 out of 10, exhibit a significant difference for both parameters, either more or less. While only one patient, no. 9, show normal latency time but different amplitude gain. Consequently, both latency time and amplitude gain are essential to perform a satisfactory diagnosis for MS disorder.

In general, the developed tool can be used by clinicians and medical physicians for the diagnosis and identification of neurological disorders.

\section{CONCLUSION}

In this work, we developed a low-cost system for saccadic measurements. The system records the raw data of gaze location and dynamic stimuli using a low-resolution webcam. We proposed an algorithmic approach to process and extract saccadic parameters, i.e., time latency, amplitude gain and peak velocity. Experimental results demonstrated that the proposed strategy is a quick, simple and efficient technique for eye tracking and saccade measurement. Future research directions might be utilizing machine learning techniques to recognize and classify normal and pathological gaze patterns. This can be used to assist clinicians and medical physicians in the diagnosis and identification of neurological disorders.

\section{REFERENCES}

[1] M. F. Land, and D. N. Lee, "Where we look when we steer," Nature, vol. 369, no. 6483, pp. 742-744, 1994.

[2] D. Noton, and L. Stark, "Scanpaths in saccadic eye movements while viewing and recognizing patterns," Vision Research, vol. 11, no. 9, pp. 929-942, 1971.

[3] P. Suppes, "Eye-movement models for arithmetic and reading performance," Eye Movements and their Role in Visual and Cognitive Processes, vol. 4, pp. 455-477, 1990.

[4] D. D. Salvucci, and J. R. Anderson, "Integrating analogical mapping and general problem solving: The path-mapping theory," Cognitive Science, vol. 25, no. 1, pp. 67-110, 2001.

[5] A. Bulling, and H. Gellersen, "Toward mobile eye-based human computer interaction," IEEE Pervasive Computing, vol. 9, no. 4, pp. 8-12, 2010.

[6] K. Rayner, "Eye movements and cognitive processes in reading, visual search, and scene perception," Studies in Visual Information Processing, vol. 6, pp. 3-22, 1995.

[7] C. Knox, "The parameters of eye movement," Lecture Notes, $<$ http://www. liv. ac. uk/ pcknox/teaching/Eymovs/params. htm>.

[8] S. B. Hutton, "Cognitive control of saccadic eye movements," Brain and Cognition, vol. 68, no. 3, pp. 327-340, 2008.

[9] M. Ibbotson, and B. Krekelberg, "Visual perception and saccadic eye movements," Current Opinion in Neurobiology, vol. 21, no. 4, pp. 553-558, 2011.

[10] P. Termsarasab, T. Thammongkolchai, J. C. Rucher, and S. J. Frucht, "The diagnostic value of saccades in movement disorder patients: a practical guide and review," Journal of Clinical Movement Disorders, vol. 2, no. 1, pp. 1-10, 2015.

[11] S. Stuart, A. Hickey, B. Galna, S. Lord, L. Rochester, and A. Godfrey, "iTrack: instrumented mobile electrooculography (EOG) eye-tracking in older adults and Parkinson's disease," Physiological Measurement, vol. 38, no. 1, pp. N16-N31, 2016.

[12] S. Stuart, S. Lord, B. Galna, and L. Rochester, "Saccade frequency response to visual cues during gait in Parkinson's disease: the selective role of attention," European Journal of Neuroscience, vol. 47, no. 7, pp. 769-778, 2018.

[13] A. T. Duchowski, "Eye Tracking Methodology," Theory and Practice, vol. 328, no. 614, pp. 2-3, 2007.

[14] A. Agarwal, D. JeevithaShree, K. S. Saluja, A. Sahay, P. Mounika, A. Sahu, R. Bhaumik, V. K. Rajendran, and P. Biswas, "Comparing two webcam-based eye gaze trackers for users with severe speech and motor impairment," Research into Design for a Connected World, pp. 641-652, 2019.

[15] L. Larsson, Event Detection in Eye-Tracking Data for Use in Applications with Dynamic Stimuli. Lund University, 2016.

[16] S. Stuart, A. Hickey, R. Vitorio, K. Welman, S. Foo, D. Keen, A. Godfrey, "Eye-tracker algorithms to detect saccades during static and dynamic tasks: a structured review," Physiological Measurement, vol. 40, no. 2, pp. 02TR01, 2019. 
[17] M. Alaqtash, H. Yu, R. Brower, A. Abdelgawad, and T. Sarkodie-Gyan, "Application of wearable sensors for human gait analysis using fuzzy computational algorithm," Engineering Applications of Artificial Intelligence, vol. 24, no. 6, pp. 1018-1025, 2011.

[18] M. Alaqtash, T. Sarkodie-Gyan, H. Yu, O. Fuentes, R. Brower, and A. Abdelgawad, "Automatic classification of pathological gait patterns using ground reaction forces and machine learning algorithms," Proc. 33rd Ann. Int. Conf. IEEE Engineering in Medicine and Biology Society (EMBC'11), Boston, MA, pp. 453-457, 2011.

[19] L. Setlak, and R. Kowalik, "Control model of a small micro-class UAV object taking into account the impact of strong wind," WSEAS Transactions on Systems and Control, vol. 14, pp. 411-418, 2019.

[20] L. Setlak, and R. Kowalik, "Control system of the multi-rotor in flight in the presence of strong wind," WSEAS Transactions on Systems and Control, vol. 14, pp. 437-444, 2019.

[21] J. Abukhait, A. Aljaafreh, and N. Al-Oudat, “A Multi-agent design of a computer player for nine men's morris board game using deep reinforcement learning," 2019 Sixth International Conference on Social Networks Analysis, Management and Security (SNAMS), Granada, Spain, pp. 489-493, 2019

[22] J. Abukhait, "Visibility estimation of road signs considering detectability factors for driver assistance systems," WSEAS Transactions on Signal Processing, vol. 12, pp. 111-117, 2016.

[23] J. Abukhait, I. Abdel-Qader, J. Oh, and O. Abudayyeh, "Road sign detection and shape recognition invariant to sign defects," 2012 IEEE International Conference on Electro/Information Technology, Indianapolis, IN, pp. 1-6, 2012.

[24] <https://github.com/jaafreh/saccadic-measurement>

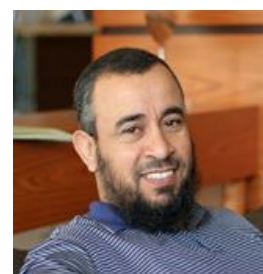

Dr. Ahmad Aljaafreh received his PhD in Computer Engineering in 2010 from Western Michigan University. In the same year, he joined Tafila Technical University in Tafila, Jordan. Currently, he is an associate professor at the Department of Communications, Electronics and Computer Engineering at Tafila Technical University. His research interests are in, AI and embedded systems.

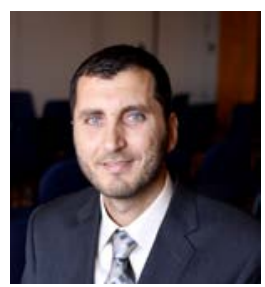

Dr. Murad Alaqtash is an assistant professor of computer engineering at Tafila Technical University. He received his $\mathrm{PhD}$ degree in computer engineering from the University of Texas at El Paso, Texas, USA in 2012. His research interests are artificial intelligence, machine learning, smart embedded systems, and wearable sensors.

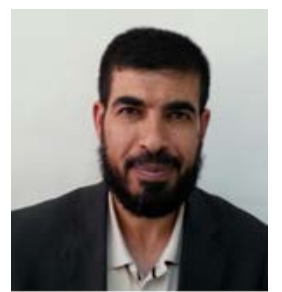

Dr. Naeem Al-Oudat received his $\mathrm{PhD}$ in Computer engineering form Iowa state university in 2012. Since 2012, He is serving as a faculty member in Communications and Computer engineering department in Tafila Technical
University. His research interests include real-time distributed systems task optimizing and scheduling, educational operating systems design.

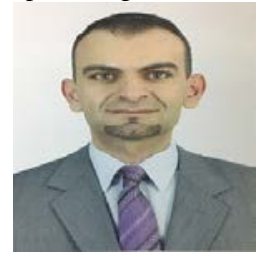

Dr. Jafar Abukhait received his BS and MS in Computer Engineering from Yarmouk University in 2005 and 2007, respectively. He received his PhD in Electrical and Computer Engineering from Western Michigan University in 2012. In 2012, he joined the faculty of Tafila Technical University as an Assistant Professor of Electrical and Computer Engineering. He got promoted to Associate Professor in 2018. His research interests are mainly in digital image processing, feature extraction, pattern recognition, and classification techniques.

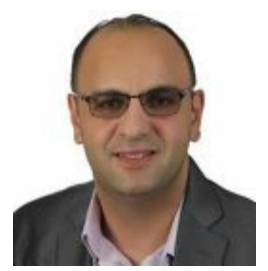

Dr. Ma'en Saleh received his Ph.D. degree in electrical and computer engineering from Western Michigan University in 2012. He joined the faculty of Tafila Technical University as an assistant professor of Electrical and Computer Engineering in 2012. He promoted to associate professor in 2018. His research interests include real-time scheduling for packet switched networks, security in VANETs, Simulating real-time networks, real-time agent-based systems, and QoS for heterogeneous networks. 